\title{
VAGUE FIELDS AND VAGUE VECTOR SPACES
}

\author{
T. Eswarlal ${ }^{1} \S$, N. Ramakrishna ${ }^{2}$, \\ ${ }^{1}$ Department of Mathematics \\ KL University \\ Vaddeswaram, 522 502, Guntur Dist. Andhra Pradesh, INDIA \\ ${ }^{2}$ Department of Mathematics \\ Mrs. A.V.N. College \\ Visakhapatnam, Andhra Pradesh, INDIA
}

\begin{abstract}
The notions of vague fields and vague vector spaces with membership and non-membership function values taking in unit interval of real numbers are introduced, which generalize the existing notions of fuzzy field, fuzzy vector spaces, and studied various properties.
\end{abstract}

AMS Subject Classification: 08A72, 20N25, 03E72

Key Words: vague set, vague fields, vague vector spaces

\section{Introduction}

The concept of fuzzy sets and fuzzy set operations was introduced by Zadeh[12]. Since then the theory of fuzzy sets developed by Zadeh and others evoked tremendous interest among researchers working in different branches of Mathematics, and it leads to various aspects of the theory and applications of fuzzy sets such as; fuzzy topological spaces, similarity relations and fuzzy order rings, algebraic properties of fuzzy sets, fuzzy measures, probability measures of fuzzy

Received: June 17, 2013

(C) 2014 Academic Publications, Ltd.

${ }^{\S}$ Correspondence author url: www.acadpubl.eu 
events, fuzzy mathematical programming, fuzzy dynamic programming and decision making on a fuzzy environment. The fuzzy set assigns to each object a single value. This single value combines the evidence for $x \in X$, and the evidence against $x \in X$, with out indicating how much there is of each. The single number tells us nothing about its accuracy. To overcome this situation, Gau and Buehrer [3] in 1993 introduced and studied Vague sets. The theory of vague sets started with of interpretting the real life problems in a betterway than the fuzzy sets do. Infact a vague set $A$ on a set $X$ is a pair $\left(t_{A}, f_{A}\right)$ where $t_{A}$ is called a membership function and $f_{A}$ is called a non-membership function are fuzzy sets on $X$ with $t_{A}(x) \leq 1-f_{A}(x)$ for all $x$ in $X$. Also the vague sets are generalization of study of fuzzy sets, because fuzzy sets $t_{A}$ is identified with pair $\left(t_{A}, 1-t_{A}\right)$.

Ranjit Biswas [8] initiated the study of vague algebra by studying the vague groups in the lines of fuzzy algebra by studying the concepts of vague groups, vague cuts, vague normal groups etc. Further RamaKrishna.N.[6,7] and EswarLal.T.[2] continued the study of vague algebra by studying the characterization of cyclic groups in terms of vague groups, vague normal groups, vague normalizer, vague centralizer, vague ideals, normal vague ideals etc. Surdarsan Nanda [9] have introduced and studied the concepts of fuzzy field and fuzzy linear spaces over the fields and established various results.

The aim of paper is study vague fields and vague vector spaces over a field. Let $(K,+,$.$) be a field, we established a condition that F$ is a vague field of $X$ if and only if $V_{F}(x-y) \geq \min \left\{V_{F}(x), V_{F}(y)\right\}$ for all $x, y \in X$ and $V_{F}\left(x . y^{-1}\right) \geq \min \left\{V_{F}(x), V_{F}(y)\right\}$ for all $x \in X, 0 \neq y \in X$. Also we introduced the notion of vague vector spaces over a field $X$ and established a necessary and sufficient condition for a vague set $F$ to be a vague vector space over a field $X$ and investigate some interesting results.

\section{Preliminaries}

We give here a review of some definitions and results which are in W.L . Gau and Buehrer [3],Hakimuddin Khan, Musheer Ahmad, and Ranjit Biswas [4], Ranjit Biswas [8].

Definition 1.1. A vague set $A$ in the universe of discourse $U$ is a pair $\left(t_{A}, f_{A}\right)$, where $t_{A}: U \rightarrow[0,1], f_{A}: U \rightarrow[0,1]$ are mappings suchthat $t_{A}(u)+$ $f_{A}(u) \leq 1$ for all $u \in U$. The functions $t_{A}$ and $f_{A}$ are called true membership function and false membership function in $[0,1]$ respectively. 
Definition 1.2. The interval $\left[t_{A}(u), 1-f_{A}(u)\right]$ is called the vague value of $u$ in $A$, and it is denoted by $V_{A}(u)$, i.e. $V_{A}(u)=\left[t_{A}(u), 1-f_{A}(u)\right]$.

Notation 1.3. Let $I[0,1]$ denotes the family of all closed subinterval of $[0,1]$. If $I_{1}=\left[a_{1}, b_{1}\right]$ and $I_{2}=\left[a_{2}, b_{2}\right]$ be two elements of $I[0,1]$,we call $I_{1} \geq I_{2} \quad$ if $a_{1} \geq a_{2}$ and $b_{1} \geq b_{2}$, with the order in $I[0,1]$ is a lattice with operations min or inf and max or sup given by

$$
\begin{aligned}
\min \left\{I_{1}, I_{2}\right\} & =\left[\min \left(a_{1}, a_{2}\right), \min \left(b_{1}, b_{2}\right)\right], \\
\max \left\{I_{1}, I_{2}\right\} & =\left[\max \left(a_{1}, a_{2}\right), \max \left(b_{1}, b_{2}\right)\right] .
\end{aligned}
$$

Definition 1.4. A vague set $A=\left(t_{A}, f_{A}\right)$ is contained in the vague set $B=\left(t_{B}, f_{B}\right), \quad A \subseteq B$ if and only if $V_{A}(x) \leq V_{B}(x)$ for all $x \in U$, i.e.

$$
t_{A}(x) \leq t_{B}(x) \text { and } f_{A}(x) \geq f_{B}(x) \text { for all } x \in U .
$$

Definition 1.5. If $A$ and $B$ are two vague sets in $U$, then the intersection $A \cap B$ is defined by $A \cap B=\left(t_{A \cap B}, f_{A \cap B}\right)$, where $V_{A \cap B}(x)=\min \left\{V_{A}(x), V_{B}(x)\right\}$ for all $x \in U$, i.e.

$$
t_{A \cap B}(x)=\min \left\{t_{A}(x), t_{B}(x)\right\}
$$

and

$$
f_{A \cap B}(x)=\max \left\{f_{A}(x), f_{B}(x)\right\} \text { for all } x \in X .
$$

Definition 1.6. If $A$ and $B$ are two vague sets in $U$, then the union $A \cup B$ is defined by $A \cup B=\left(t_{A \cup B}, f_{A \cup B}\right)$ where $V_{A \cup B}(x)=\max \left\{V_{A}(x), V_{B}(x)\right\}$ for all $x \in U$.

$$
\text { i.e., } t_{A \cup B}(x)=\max \left\{t_{A}(x), t_{B}(x)\right\}
$$

and

$$
f_{A \cup B}(x)=\max \left\{f_{A}(x), f_{B}(x)\right\} \text { forall } x \in X .
$$

Definition 1.7. A vague set $A=\left(t_{A}, f_{A}\right)$ of a set $U$ with $t_{A}(x)=0$ and $f_{A}(x)=1$ for all $x$ in $U$ is called the Zero vague set, denoted by $\mathbf{0}$.

Definition 1.8. A vague set $A=\left(t_{A}, f_{A}\right)$ of a set $U$ with $t_{A}(x)=1$ and $f_{A}(x)=0$ for all $x$ in $U$ is called the Unit vague set, denoted by $\mathbf{1}$. 
More generally, $L=[0,1]$ is complete lattice, then for a family of vague sets $A=\left\{A_{i} / i \in I\right\}$, the union $C=\bigcup_{i \in I} A_{i}$ and the intersection $D=\bigcap_{i \in I} A_{i}$ are defined by

$$
\begin{gathered}
V_{C}(x)=\sup _{i \in I} V_{A_{i}}(x) \text {.i.e., } t_{C}(x)=\sup _{i \in I} t_{A_{i}}(x) a n d f_{C}(x)=\inf _{i \in I} f_{A_{i}}(x) \\
\text { and } V_{D}(x)=\inf _{i \in I} V_{A_{i}}(x) \text {.i.e., } t_{D}(x)=\inf _{i \in I} t_{A_{i}}(x) a n d f_{D}(x)=\sup _{i \in I} f_{A_{i}}(x)
\end{gathered}
$$

for all $x \in U$.

Definition 1.9. Let $f$ be a mapping from a set $X$ into set $Y$. Let $B$ be a vague set in $Y$; Then the inverse image of $B, f^{-1}[B]$, is the vague set in $X$ by

$$
V_{f^{-1}(B)}(x)=V_{B}(f(x)) \text { for all } x \in X \text {. }
$$

Definition 1.10. Let $A$ be a vague set in $X$ with vague value $V_{A}$. Then the image of $A, f[A]$ is the vague set in $Y$ with vague value $V_{A}$ defined by

$$
V_{f[A]}(y)= \begin{cases}\sup _{z \in f^{-1}(y)} & \text { if } f^{-1}(y) \neq \phi \\ \mathbf{0} & \text { otherwise }\end{cases}
$$

for all $y \in Y$, where $f^{-1}(y)=\{x / f(x)=y\}$.

Definition 1.11. Let $(G, *)$ be a group. A vague set $A$ of $G$ is called vague group of $G$ if for all $x, y$ in $G, V_{A}(x y) \geq \min \left\{V_{A}(x), V_{A}(y)\right\}$ and $V_{A}\left(x^{-1}\right) \geq$ $V_{A}(x)$ for all $x$ in $G$,i.e.

$$
t_{A}(x y) \geq \min \left\{t_{A}(x), t_{A}(y)\right\} \text { and } f_{A}(x y) \leq \max \left\{f_{A}(x), f_{A}(y)\right\},
$$

and

$$
t_{A}\left(x^{-1}\right) \geq t_{A}(x), \quad f_{A}\left(x^{-1}\right) \leq f_{A}(x) .
$$

Here the element $x y$ stands for $x * y$.

\section{Vague Fields}

We now introduce the concept of vague fields.

Definition 2.1. Let $X$ be a field and $F$ be a vague set of $X$. Then $F$ is a vague field of $X$ iff the following conditions are satisfied: 
(i) $V_{F}(x+y) \geq \min \left\{V_{F}(x), V_{F}(y)\right\}$ for all $x, y \in X$;

(ii) $V_{F}(-x) \geq V_{F}(x)$ for all $x \in X$;

(iii) $V_{F}(x y) \geq \min \left\{V_{F}(x), V_{F}(y)\right\}$ for all $x, y \in X$;

(iv) $V_{F}\left(x^{-1}\right) \geq V_{F}(x)$ for all $0 \neq x \in X$.

Now we have the following examples.

Example 2.2. Let $X=\left(\mathbb{Z}_{3}=\{\overline{0}, \overline{1}, \overline{2}\}\right)$ be the field under residue classes of addition and multiplication modulo-3. A vague set $A=\left(t_{A}, f_{A}\right)$ of $X$ defined as $t_{A}: X \rightarrow[0,1]$ by

$$
t_{A}(x)= \begin{cases}1 & \text { for } x=\overline{0} \\ 0.8 & \text { for } x=\overline{1}, \overline{2}\end{cases}
$$

and $f_{A}: X \rightarrow[0,1]$ by

$$
f_{A}(x)= \begin{cases}0 & \text { for } x=\overline{0} \\ 0.2 & \text { for } x=\overline{1}, \overline{2}\end{cases}
$$

is a vague field of $X$.

Example 2.3. Let $X=\left(\mathbb{Z}_{5}=\{\overline{0}, \overline{1}, \overline{2}, \overline{3}, \overline{4}\}\right)$ be the field under residue classes of addition and multiplication modulo-5. A vague set $A=\left(t_{A}, f_{A}\right)$ of $X$ defined as $t_{A}: X \rightarrow[0,1]$ by

$$
t_{A}(x)= \begin{cases}1 & \text { for } x=\overline{0} \\ 0.8 & \text { for } x=\overline{2}, \overline{3} \\ 0 & \text { for } x=\overline{1}, \overline{4}\end{cases}
$$

and $f_{A}: X \rightarrow[0,1]$ by

$$
f_{A}(x)= \begin{cases}0 & \text { for } x=\overline{0} \\ 0.2 & \text { for } x=\overline{2}, \overline{3} \\ 1 & \text { for } x=\overline{1}, \overline{4}\end{cases}
$$

is a vague field of $X$.

Example 2.4. Let $\mathbb{R}=$ The set of real numbers. Then $\mathbb{R}=(\mathbb{R},+, \cdot)$ is a field.

Define a vague set $A=\left(t_{A}, f_{A}\right)$ of $\mathbb{R}$ by $t_{A}: \mathbb{R} \rightarrow[0,1] b y$

$$
t_{A}(x)= \begin{cases}1 & \text { for } x \in\{0,1\} \\ 0.8 & \text { for xisrationalnumberotherthan } 0 \text { and } 1 \\ 0 & \text { for xisirrational }\end{cases}
$$


and $f_{A}: \mathbb{R} \rightarrow[0,1]$ by

$$
f_{A}(x)= \begin{cases}0 & \text { for } x \in\{0,1\} \\ 0.2 & \text { for xisrationalnumberotherthan0and } 1 \\ 1 & \text { for xisirrational }\end{cases}
$$

Then $A$ is vague field of $\mathbb{R}$.

We have the following proposition.

Proposition 2.5. Let $X$ be a field and $F$ be a vague set of $X$. Then $F$ is a vague field of $X$ iff: (i) $V_{F}(x-y) \geq \min \left\{V_{F}(x), V_{F}(y)\right\}$ forall $x, y \in X$;

(ii) $V_{F}\left(x y^{-1}\right) \geq \min \left\{V_{F}(x), V_{F}(y)\right\}$ forall $x \in X, 0 \neq y \in X$.

Proof. Proof is obvious by definition.

Corollary 2.6. Let $X$ be a field and $F$ be a vague field of $X$. Then we have:

(i) $V_{F}(0) \geq V_{F}(x)$ forall $x \in X$;

(ii) $V_{F}(1) \geq V_{F}(x)$ forall $\neq \neq x \in X$.

Proposition 2.7 : Let $X$ and $Y$ be fields and $f$ be a homomorphism from $X$ into $Y$. Let $F$ be a vague field of $Y$, then the inverse image $f^{-1}[F]$ of $F$ is a vague field of $X$.

Proof. For all $x, y \in X \quad V_{f^{-1}[F]}(x-y)=V_{F}(f(x-y))$

$$
\begin{gathered}
=V_{F}(f(x)-f(y)) \\
\geq \min \left\{V_{F}(f(x)), V_{F}(f(y))\right\} \\
=\min \left\{V_{f^{-1}[F]}(x), V_{f^{-1}[F]}(y)\right\} .
\end{gathered}
$$

Also, for all $x \in X, 0 \neq y \in X$, $V_{f^{-1}[F]}\left(x y^{-1}\right)=V_{F}\left(f\left(x y^{-1}\right)\right)$

$$
\begin{gathered}
=V_{F}\left(f(x) \cdot f\left(y^{-1}\right)\right) \\
\geq \min \left\{V_{F}(f(x)), V_{F}(f(y))\right\} \\
=\min \left\{V_{f^{-1}[F]}(x), V_{f^{-1}[F]}(y)\right\} .
\end{gathered}
$$

This completes the proof. 
For image's of vague sets, we have the following property [4].

A vague set $A$ of $X$ is said to have the Sup. property if for any subset $T \subset X$, there exists $x_{0} \in T$ such that $V_{A}\left(x_{0}\right)=\operatorname{Sup}_{x \in T} V_{A}(x)$.

Immediately we have the following.

Proposition 2.8. Let $X$ and $Y$ be fields and $f$ be a homomorphism of $X$ into $Y$. Let $F$ be a vague field of $X$, that has the Sup. property. Then the image $f[F]$ of $F$ is a vague field in $Y$.

Proof. Let $\alpha, \beta \in Y$, if either $f^{-1}(\alpha)$ or $f^{-1}(\beta)$ is empty, then the properties in proposition [2.5] is trivially satisfied. Suppose neither $f^{-1}(\alpha)$ nor $f^{-1}(\beta)$ is empty.

Let $p_{0} \in f^{-1}(\alpha), \quad q_{0} \in f^{-1}(\beta)$ be such that

$$
V_{F}\left(p_{0}\right)=\sup _{x \in f^{-1}(\alpha)} V_{F}(x), V_{F}\left(q_{0}\right)=\sup _{y \in f^{-1}(\beta)} V_{F}(y)
$$

Then $V_{f[F]}(\alpha-\beta)=\sup _{r \in f^{-1}(\alpha-\beta)} V_{F}(r)$ [ by definition 1.10 ]

$$
\begin{aligned}
& \geq \min \left\{V_{F}\left(p_{0}\right), V_{F}\left(q_{0}\right)\right\} \\
= & \min \left\{V_{f[F]}(\alpha), V_{f[F]}(\beta)\right\} .
\end{aligned}
$$

Also, for $\alpha \in Y$ and $0 \neq \beta \in Y$

$V_{f[F]}\left(\alpha \beta^{-1}\right)=\sup _{\theta \in f^{-1}\left(\alpha \beta^{-1}\right)} V_{F}(\theta)$

$$
\geq \min \left\{V_{f[F]}(\alpha), V_{f[F]}(\beta)\right\}
$$

This completes the proof.

Proposition 2.9. If $F$ is a vague field of a field $X$, then:

(i) $V_{F}(-x)=V_{F}(x)$ for all $x \in X$;

(ii) $V_{F}\left(x^{-1}\right)=V_{F}(x)$ for all $0 \neq x \in X$.

Proof. By Proposition [2.5], we have $V_{F}(-x) \geq V_{F}(x)$ for all $x \in X$, also we have $V_{F}(-(-x)) \geq V_{F}(-x)$ i.e., $V_{F}(x) \geq V_{F}(-x)$. Hence condition $(i)$ is proved. Similarly we can prove the condition $(i i)$ for $0 \neq x \in X$.

Proposition 2.10. The intersection of a family of vague fields is a vague field. 
Proof. Since $L=[0,1]$ is a complete lattice, and let $\left\{F_{i} / i \in I\right\}$ be a family of vague fields; and $F=\bigcap_{i \in I} F_{i}$, then

$$
\begin{gathered}
V_{F}(x-y)=\inf _{i \in I} V_{F_{i}}(x-y) \\
\geq \inf _{i \in I}\left\{\min \left\{V_{F_{i}}(x), V_{F_{i}}(y)\right\}\right\} \\
=\min \left\{\inf _{i \in I} V_{F_{i}}(x), \inf _{i \in I} V_{F_{i}}(y)\right\} \\
=\min \left\{V_{F}(x), V_{F}(y)\right\} .
\end{gathered}
$$

Also

$$
\begin{gathered}
V_{F}\left(x y^{-1}\right)=\inf _{i \in I} V_{F_{i}}\left(x y^{-1}\right) \\
\geq \inf _{i \in I}\left\{\min \left\{V_{F_{i}}(x), V_{F_{i}}(y)\right\}\right\} \\
=\min \left\{\inf _{i \in I} V_{F_{i}}(x), \inf _{i \in I} V_{F_{i}}(y)\right\} \\
=\min \left\{V_{F}(x), V_{F}(y)\right\} .
\end{gathered}
$$

This completes the proof.

\section{Vague Vector Spaces}

We now introduce the concept of vague vector spaces which is the generalized version of fuzzy vector spaces [5] taking the vague values in complete lattice $L=[0,1]$.

Definition 3.1 : Let $X$ be a field and let $Y$ be a vector space over $X$, and $A$ is a vague set of $Y$. Then $A$ is a vague vector space of $Y$ if the following properties are hold;

$$
\begin{gathered}
(i) V_{A}(x+y) \geq \min \left\{V_{A}(x), V_{A}(y)\right\} \text { for all } x, y \in Y \\
(i i) V_{A}(\lambda x) \geq V_{A}(x) \text { forall } \lambda \text { inXandall } x \in Y \\
(\text { iii }) V_{A}(0)=1
\end{gathered}
$$

We have the following proposition.

Proposition 3.2. Let $Y$ be a vector space over the field $X$ and $A$ is a vague set of $Y$. The $A$ is vague vector space of $Y$ iff

$$
V_{A}(\lambda x+\mu y) \geq \min \left\{V_{A}(x), V_{A}(y)\right\} \text { for allx, } y \in Y ; \lambda, \mu \in X \text {. }
$$


Proof. Let $A$ be a vague vector space of $Y$. Then we have $V_{A}(\lambda x) \geq$ $V_{A}(x)$ and $V_{A}(\mu y) \geq V_{A}(y)$ forall $x \in Y$ and $\lambda \in X$. Hence $V_{A}(\lambda x+\mu y) \geq$ $\min \left\{V_{A}(x), V_{A}(y)\right\}$.

Conversely, if $\lambda=\mu=1$, then $V_{A}(x+y) \geq \min \left\{V_{A}(x), V_{A}(y)\right\}$ and also if $\mu=0$, then $V_{A}(\lambda x)=V_{A}(\lambda x+0 . x) \geq \min \left\{V_{A}(x), V_{A}(x)\right\}$. Hence $V_{A}(\lambda x) \geq$ $V_{A}(x)$ foralliin $X$ andall $x \in Y$.

Lemma 3.3. The intersection of a family of vague vector spaces is a vague vector space.

Proof. Let $\left\{A_{i} / i \in I\right\}$ be a family of vague vector spaces, and let $A=$ $\cap_{i \in I} A_{i}$, then

$$
\begin{gathered}
V_{A}(x+y)=\inf _{i \in I} V_{A_{i}}(x+y) \\
\geq \inf _{i \in I}\left\{\min \left\{V_{A_{i}}(x), V_{A_{i}}(y)\right\}\right\} \\
=\min \left\{\inf _{i \in I} V_{A_{i}}(x), \inf _{i \in I} V_{A_{i}}(y)\right\} \\
=\min \left\{V_{A}(x), V_{A}(y)\right\} . \\
A l s o V_{A}(\lambda x)=\inf _{i \in I} V_{A_{i}}(\lambda x) \\
\geq \inf _{i \in I}\left\{\min \left\{V_{A_{i}}(x), V_{A_{i}}(x)\right\}\right\} \\
=\min \left\{\inf _{i \in I} V_{A_{i}}(x)\right\} \\
=\min \left\{V_{A}(x)\right\} \\
=V_{A}(x) .
\end{gathered}
$$

This completes the proof.

Proposition 3.4. Let $Y$ and $Z$ be a vector space over a field $X$, and $f$ be a linear transformation of $Y$ into $Z$. Let $W$ be a vague vector space of $Z$. Then the inverse image $f^{-1}(W)$ of $W$ is a vague vector space of $Y$.

Proof. For all $x, y \in Y$ and $\lambda, \mu \in X$, then

$$
\begin{aligned}
& V_{f^{-1}(W)}(\lambda x+\mu y)=V_{W}(f(\lambda x+\mu y)) \\
&=V_{W}(\lambda f(x)+\mu f(y)) \\
& \geq \min \left\{V_{W}(f(x)), V_{W}(f(y))\right\} \\
&=\min \left\{V_{f^{-1}(W)}(x), V_{f^{-1}(W)}(y)\right\} .
\end{aligned}
$$

This completes the proof. 
Theorem 3.5. Let $Y$ and $Z$ be a vector space over a field $X$, and $f$ be a linear transformation of $Y$ into $Z$. Let $A$ be a vague vector space of $Y$, that has the Sup. Property. Then image $f[A]$ of $A$ is a vague vector space of $Z$.

Proof. Let $\alpha, \beta \in Z$, if either $f^{-1}(\alpha)$ or $f^{-1}(\beta)$ is empty then the inequality of Proposition [3.2] is satisfied. Suppose neither $f^{-1}(\alpha)$ nor $f^{-1}(\beta)$ is empty.

Let $p_{0} \in f^{-1}(\alpha) ; \quad q_{0} \in f^{-1}(\beta)$

$$
\begin{gathered}
V_{A}\left(p_{0}\right)=\sup _{x \in f^{-1}(\alpha)} V_{A}(x) ; V_{A}\left(q_{0}\right)=\sup _{y \in f^{-1}(\beta)} V_{A}(y) \\
\text { Then } V_{f[A]}(\lambda \alpha+\mu \beta)=\sup _{r \in f^{-1}(\lambda \alpha+\mu \beta)} V_{A}(r) \\
\geq \min \left\{V_{A}\left(p_{0}\right), V_{A}\left(q_{0}\right)\right\} \\
=\min \left\{V_{f[A]}(\alpha), V_{f[A]}(\beta)\right\} .
\end{gathered}
$$

This completes the proof.

Proposition 3.6. If $A$ is a vague vector space of a vector space $Y$ over a field $X$, then $V_{A}(\lambda x)=V_{A}(x)$ for all $x \in Y$ and all $0 \neq \lambda \in X$.

Proof. For all $x \in Y$, we have $V_{A}(\lambda x) \geq V_{A}(x)$. For $0 \neq \lambda \in X$, and all $x \in Y$, we have

$$
V_{A}(x)=V_{A}\left(\lambda^{-1} \lambda x\right) \geq V_{A}(\lambda x) .
$$

Hence $V_{A}(\lambda x)=V_{A}(x)$ for all $x \in Y \& 0 \neq \lambda \in X$.

This completes the proof.

\section{Conclusion}

In this paper, the concept of vague vector spaces has been introduced and it is expected that several results from linear algebra and functional analysis can be extended to the concept of vague sets. Particularly, it is hoped that, the concept of vague vector spaces will give rise to the notions like vague linear spaces and vague normal linear spaces. 


\section{Acknowledgments}

The authors are grateful to Prof. K.L.N. Swamy for his valuable suggestions and discussions on this work.

\section{References}

[1] P.B. Bhattacharya, S.K. Jain, S.R. Nagpaul, Basic Abstract Algebra, Cambridge University Press, 1995.

[2] Lal.T. Eswar, Vague ideals and normal vague ideals in semirings, International Journal of Computational Coginition, 6, No. 3 (2008).

[3] W.L. Gau, D.J. Buehrer, Vague sets, IEEE Transactions on Systems, Man. and Cybernetics, 23 (1993), 610-614.

[4] Hakimuddin Khan, Musheer Ahmad, Ranjit Biswas, On vague groups, International Journal of Computational Coginition, 5, No. 1 (2007).

[5] A.K. Katsaras, D.B. Liu, Fuzzy vector spaces and fuzzy topological vector spaces, J. Math. Anal. Appl., 58 (1977), 135-144.

[6] N. Ramakrishna, Vague normal groups, International Journal of Computational Coginition, 6, No. 2 (2008).

[7] N. Ramakrishna, A characterization of cyclic groups in terms of vague groups, International Journal of Computational Coginition, 6, No. 2 (2008).

[8] Ranjit Biswas, Vague groups, International Journal of Computational Coginition, 4, No. 2 (2006).

[9] Sudarsan Nanda, Fuzzy fields and fuzzy linear spaces, Fuzzy Sets and Systems, 19 (1986), 89-94.

[10] Wang-Jin Liu, Fuzzy invariant subgroups and Fuzzy ideals, Fuzzy Sets and Systems, 8 (1982), 133-139.

[11] Wang-ming Wu, Normal fuzzy groups, Fuzzy Math., 1 (1981), 21-30.

[12] L.A. Zadeh, Fuzzy sets, Inform. and Control, 8 (1965), 338-353. 
